Supplement of Biogeosciences, 14, 1021-1038, 2017

http://www.biogeosciences.net/14/1021/2017/

doi:10.5194/bg-14-1021-2017-supplement

(C) Author(s) 2017. CC Attribution 3.0 License.

(c) (i)

Supplement of

\title{
Quantification of multiple simultaneously occurring nitrogen flows in the euphotic ocean
}

Min Nina Xu et al.

Correspondence to: Shuh-Ji Kao (sjkao@xmu.edu.cn)

The copyright of individual parts of the supplement might differ from the CC-BY 3.0 licence. 
Figure S1. Structure of the equation based STELLA box model (Version 9.14) for the low nutrient case. Square represents pool. Pools of $\mathrm{NH}_{4}{ }^{+}, \mathrm{NO}_{\mathrm{x}}{ }^{-}$and $\mathrm{PN}$ were separated into two modules (i.e., ${ }^{14} \mathrm{~N}$ and ${ }^{15} \mathrm{~N}$ ). Faucet $(\mathrm{Fi}, \mathrm{i}=1 \sim 6$, was for $\mathrm{N}$ process rate ) stands for regulator controlled by associated arrows determined by rate constant $(\mathrm{ki}, \mathrm{i}=1 \sim 6)$ and reactant concentration. Fractionation between ${ }^{14} \mathrm{~N}$ and ${ }^{15} \mathrm{~N}$ was not considered, thus, ki is the same for both ${ }^{14} \mathrm{~N}$ and ${ }^{15} \mathrm{~N}$. The output, $\mathrm{r}$, stands for the ratio of ${ }^{15} \mathrm{~N}$ to ${ }^{14} \mathrm{~N}+{ }^{15} \mathrm{~N}$ derived from instantaneous ${ }^{15} \mathrm{~N}$ and ${ }^{14} \mathrm{~N}$ amounts in pools. Exported equations were shown below.

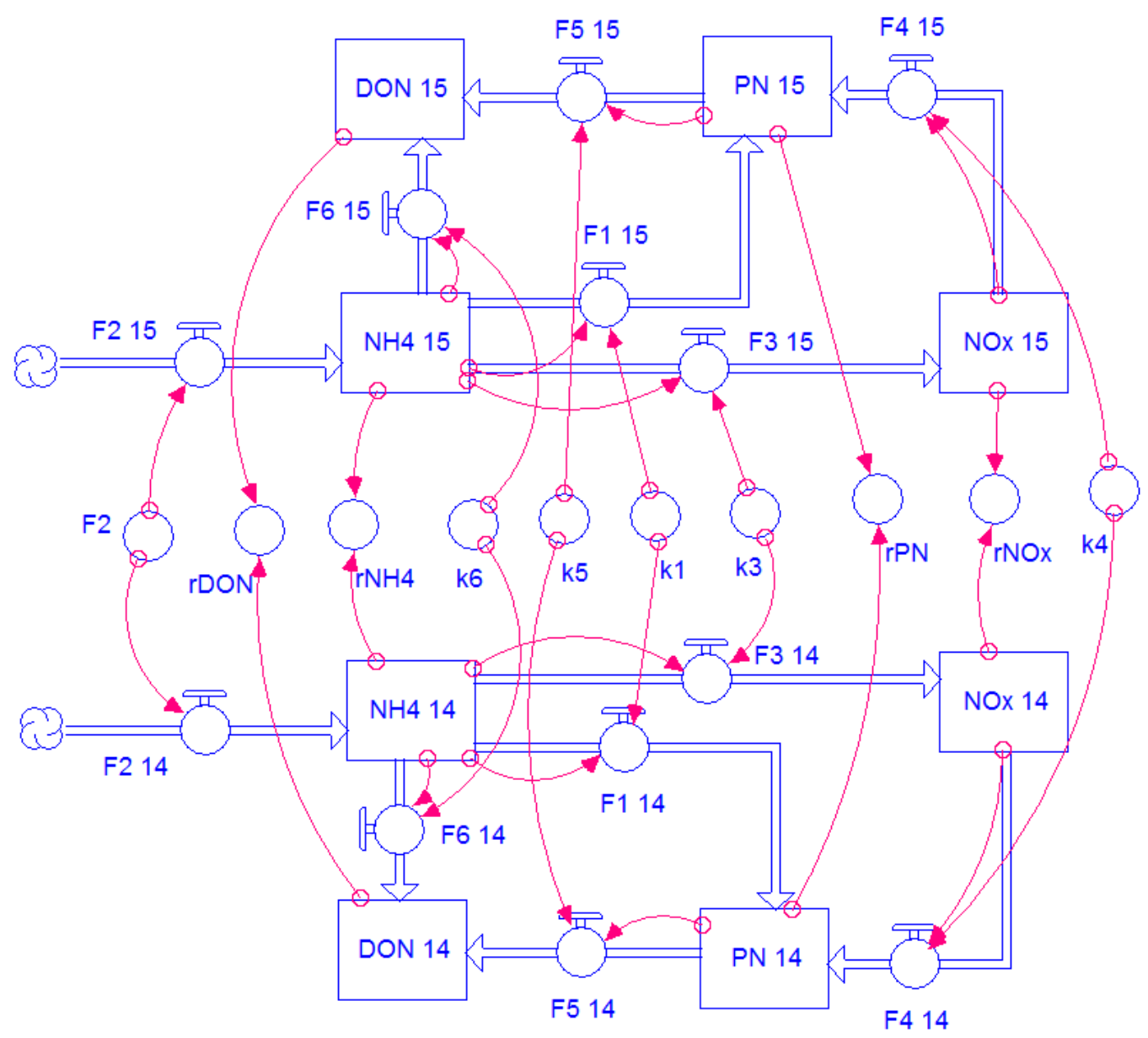

Low nutrient case Equations:

DON_14 $(\mathrm{t})=$ DON_14 $(\mathrm{t}-\mathrm{dt})+\left(\mathrm{F} 6 \_14+\mathrm{F} 5 \_14\right) * \mathrm{dt}$

INIT DON_14 $=5376.4$

INFLOWS:

F6_14 = k6*NH4_14

F5_14 $=$ k5 5 PN_14

DON_15(t) $=$ DON_ $15(\mathrm{t}-\mathrm{dt})+\left(\mathrm{F} 5 \_15+\mathrm{F} 6 \_15\right) * \mathrm{dt}$

INIT DON $15=19.8$

INFLOWS:

F5 $15=$ PN_15*k5 
F6 $\_15=\mathrm{NH} 4 \_15 * \mathrm{k} 6$

$\mathrm{NH} 4 \_14(\mathrm{t})=\overline{\mathrm{N}} \mathrm{H} 4 \_14(\mathrm{t}-\mathrm{dt})+\left(\mathrm{F} 2 \_14-\mathrm{F} 6 \_14-\mathrm{F} 1 \_14-\mathrm{F} 3 \_14\right) * \mathrm{dt}$

INIT NH4_14 = $11 \overline{2} .9$

INFLOWS:

F2_14 = F2*(1-0.00366)

OUTFLOWS:

F6_14 = k6*NH4_14

$\mathrm{F} 1 \_14=\mathrm{k} 1 * \mathrm{NH} 4 \_14$

F3_ $14=\mathrm{k} 3 * \mathrm{NH} 4 \_14$

$\mathrm{NH} 4 \_15(\mathrm{t})=\mathrm{NH} 4 \_15(\mathrm{t}-\mathrm{dt})+\left(\mathrm{F} 2 \_15-\mathrm{F} 3 \_15-\mathrm{F} 1 \_15-\mathrm{F} 6 \_15\right) * \mathrm{dt}$

INIT NH4 $15=29.8$

INFLOWS:

F2_15 = F2*0.00366

OUTFLOWS:

F3_15 = NH4_15*k3

$\mathrm{F} 1-15=\mathrm{NH} 4-15 * \mathrm{k} 1$

F6 $15=\mathrm{NH} 4 \_15 * \mathrm{k} 6$

NOx_14 $(\mathrm{t})=\overline{\mathrm{NOx}} \_14(\mathrm{t}-\mathrm{dt})+\left(\mathrm{F} 3 \_14-\mathrm{F} 4 \_14\right) * \mathrm{dt}$

INIT NOx_14 $=518.8$

INFLOWS:

F3_14 = k3*NH4_14

OUTFLOWS:

F4_14 = k4*NOx_14

NOx_15(t) $=$ NOx_15(t - dt $)+\left(F 3 \_15-F 4 \_15\right) * d t$

INIT NOx_15 $=1.9$

INFLOWS:

F3_15 = NH4_15*k3

OUTFLOWS:

F4 $15=$ NOx $15 * \mathrm{k} 4$

PN_14 $(\mathrm{t})=\mathrm{PN} \_14(\mathrm{t}-\mathrm{dt})+\left(\mathrm{F} 4 \_14+\mathrm{F} 1 \_14-\mathrm{F} 5 \_14\right) * \mathrm{dt}$

INIT PN_14 $=435.1$

INFLOWS:

F4 $14=\mathrm{k} 4 * \mathrm{NOx} 14$

$\mathrm{F} 1 \_14=\mathrm{k} 1 * \mathrm{NH} 4 \_14$

OUTFLOWS:

F5_14 = k5*PN_14

PN_15(t) $=$ PN_15 $(\mathrm{t}-\mathrm{dt})+\left(\mathrm{F} 4 \_15+\mathrm{F} 1 \_15-\mathrm{F} 5 \_15\right) * \mathrm{dt}$

INIT PN_15 $=1.7$

INFLOWS:

F4 $15=$ NOx $15 * \mathrm{k} 4$

F1_15 = NH4_15*k1

OUTFLOWS:

F5_15 = PN_15*k5

$\mathrm{rDON}=\mathrm{DON} 15 /(\mathrm{DON} 14+\mathrm{DON} 15)$

$\mathrm{rNH} 4=\mathrm{NH} 4-15 /\left(\mathrm{NH} 4 \_-14+\mathrm{NH} 4 \_15\right)$

$\mathrm{rNOx}=\mathrm{NOx} \_15 /\left(\mathrm{NOx}_{-} 14+\mathrm{NOx} \mathrm{x}_{-} 15\right)$

$\mathrm{rPN}=$ PN_15/(PN_14+PN_15) 
Figure S2. The STELLA model (Version 9.14) for high nutrient case. The Fi $(i=1 \sim 8)$ indicates rate of the relevant $\mathrm{N}$ process controlled by multiplying rate constant $(\mathrm{ki}, \mathrm{i}=1 \sim 8)$ with substrate concentration. Similar to low nutrient case, $\mathrm{NH}_{4}{ }^{+}, \mathrm{NO}_{2}{ }^{-}, \mathrm{NO}_{3}{ }^{-}$and $\mathrm{PN}$ pools were separated into two modules (i.e., ${ }^{14} \mathrm{~N}$ and ${ }^{15} \mathrm{~N}$ ). Exported equations were shown below the figure.

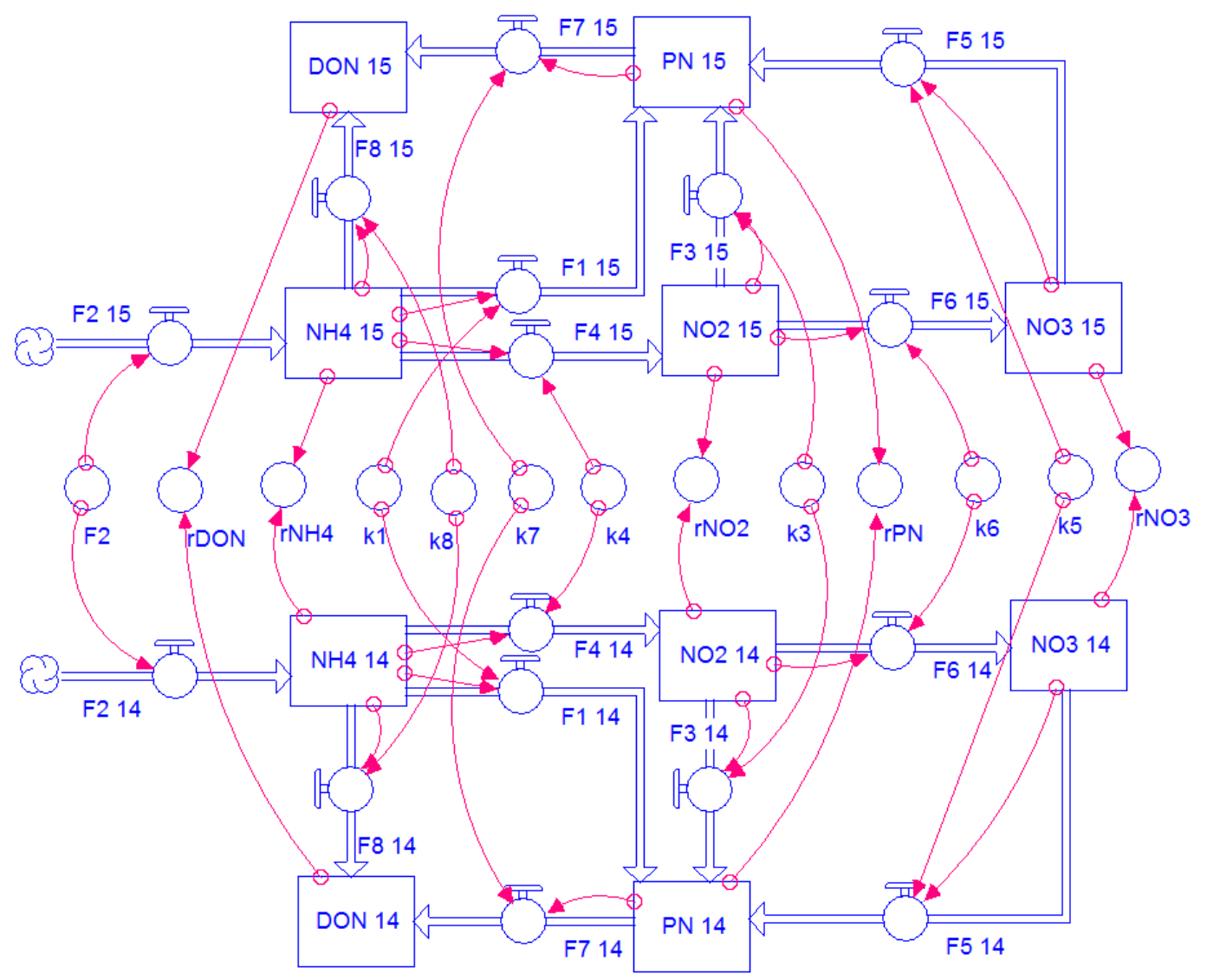

High nutrient case equations (e.g. 80\%sPAR case):

DON_14 $(\mathrm{t})=$ DON_14 $(\mathrm{t}-\mathrm{dt})+\left(\mathrm{F} 8 \_14+\mathrm{F} 7 \_14\right) * \mathrm{dt}$

INIT DON_14 $=17.39$

INFLOWS:

F8_14 $=\mathrm{k} 8 * \mathrm{NH} 4 \_14$

F7 $14=\mathrm{k} 7 * \mathrm{PN} \_14$

DON_15 $(\mathrm{t})=\overline{\mathrm{DON}} \_15(\mathrm{t}-\mathrm{dt})+\left(\mathrm{F} 7 \_15+\mathrm{F} 8 \_15\right) * \mathrm{dt}$

INIT DON_15 $=0.064$

INFLOWS:

F7 $15=\mathrm{k} 7 * \mathrm{PN} 15$

$\mathrm{F} 8 \_15=\mathrm{k} 8 * \mathrm{NH} 4 \_15$

$\mathrm{NH} 4 \_14(\mathrm{t})=\mathrm{NH} 4 \_14(\mathrm{t}-\mathrm{dt})+\left(\mathrm{F} 2 \_14-\mathrm{F} 8 \_14-\mathrm{F} 1 \_14-\mathrm{F} 4 \_14\right) * \mathrm{dt}$

INIT NH4 $14=25.54$

INFLOWS:

F2_14 = F2*(1-0.00366)

OUTFLOWS:

$\mathrm{F} 8 \_14=\mathrm{k} 8 * \mathrm{NH} 4 \_14$

$\mathrm{F} 1 \_14=\mathrm{k} 1 * \mathrm{NH} 4 \_14$ 


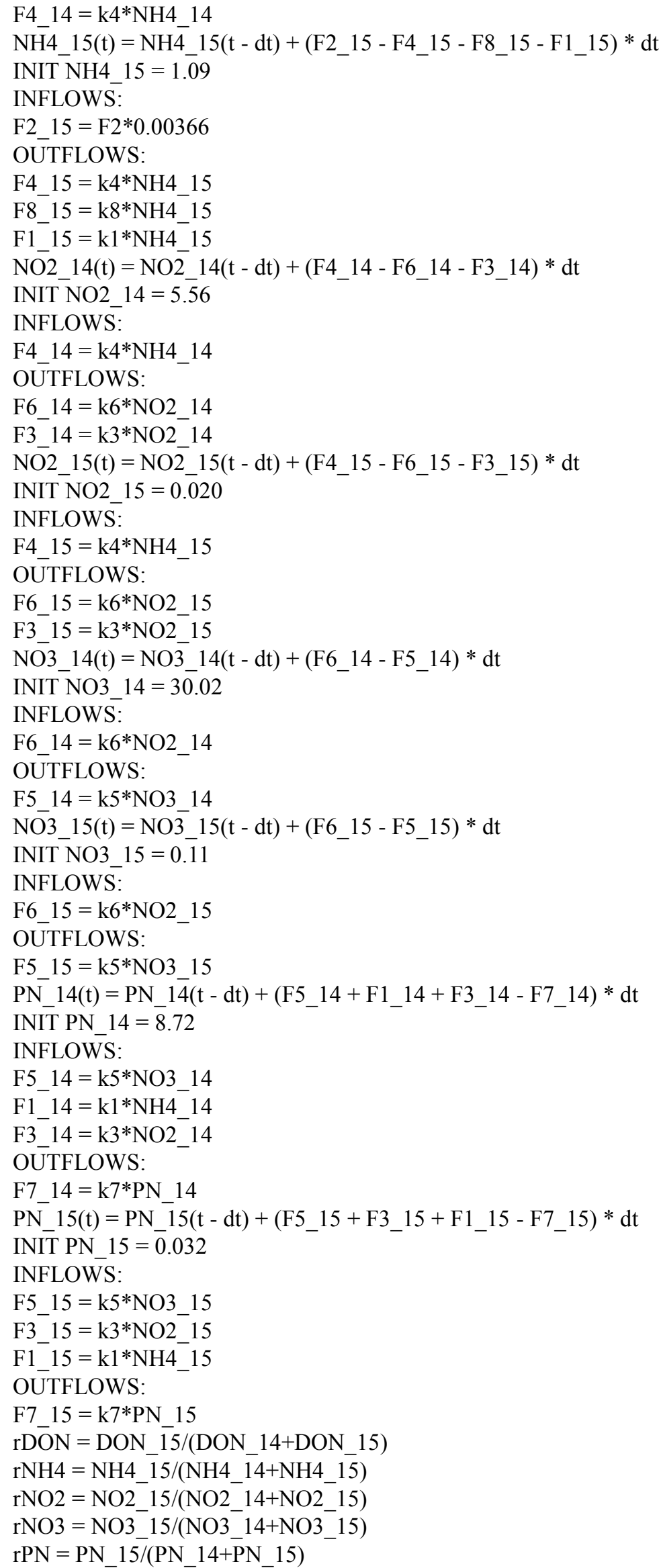


Figure S3. The temporal variations of pico-eukaryote and Synechococcus in the low-nutrient incubations.

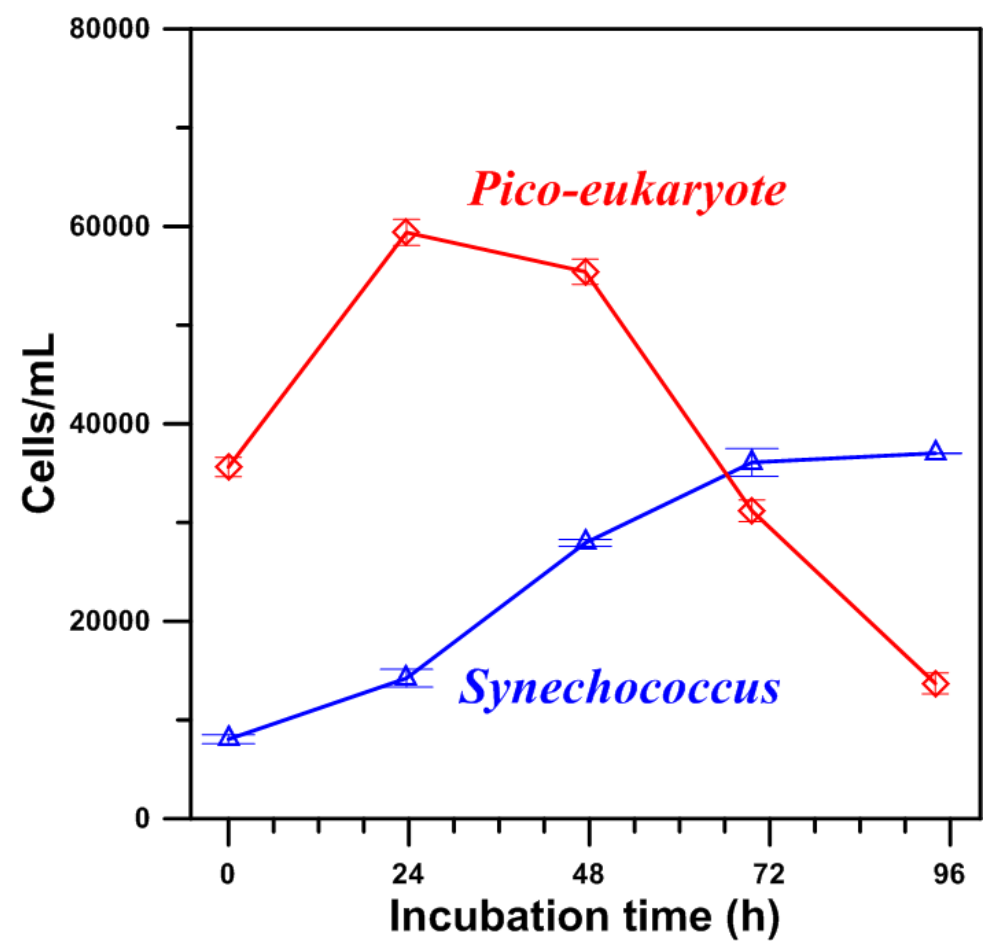

